\title{
$\$$ Research Square

\section{Automatic Phase Identification of Earthquake Based on the UBDN Deep Network}

\section{Jianxian Cai}

Institute of Disaster Prevention

Xun Dai

Institute of Disaster Prevention

Gao zhitao ( $\nabla$ gzt@cidp.edu.cn )

Institute of Disaster Prevention https://orcid.org/0000-0002-7667-3370

\section{Yan Shi}

Institute of Disaster Prevention

\section{Full paper}

Keywords: U-net, Bidirectional Long Short Term Memory, Phase identification, Wenchuan aftershocks

Posted Date: April 29th, 2021

DOl: https://doi.org/10.21203/rs.3.rs-417923/v1

License: (c) (i) This work is licensed under a Creative Commons Attribution 4.0 International License. Read Full License

Version of Record: A version of this preprint was published at Journal of Intelligent \& Fuzzy Systems on December 3rd, 2021. See the published version at https://doi.org/10.3233/JIFS-211792. 
1 Automatic phase identification of earthquake based on the

3 Author \#1: Jianxian Cai, Institute of Disaster Prevention, jianxian.cai@ hotmail.com

4 Author \#2: Xun Dai, Institute of Disaster Prevention, daixun0615@qq.com

5 Corresponding Author \#3: Zhitao Gao, Institute of Disaster Prevention,

6 e228319@163.com

7 Author \#4: Yan Shi, Institute of Disaster Prevention, windsor33@163.com

8

9

10

11

12

13

14

15

16

17

18

19

20 


\section{Abstract}

23 Seismic data obtained from seismic stations are the major source of the information

24 used to forecast earthquakes. With the growth in the number of seismic stations, the 25 size of the dataset has also increased. Traditionally, STA/LTA and AIC method have 26 been applied to process seismic data. However, the enormous size of the dataset reduces 27 accuracy and increases the rate of missed detection of the $\mathrm{P}$ and $\mathrm{S}$ wave phase when 28 using these traditional methods. To tackle these issues, we introduce the novel U-net29 Bidirectional Long-Term Memory Deep Network (UBDN) which can automatically 30 and accurately identify the $\mathrm{P}$ and $\mathrm{S}$ wave phases from seismic data. The U-net based

31 UBDN strongly maintains the U-net's high accuracy in edge detection for extracting 32 seismic phase features. Meanwhile, it also reduces the missed detection rate by applying 33 the Bidirectional Long Short-Term Memory (Bi-LSTM) mode that processes timing 34 signals to establish the relationship between seismic phase features. Experimental 35 results using the Stanford University seismic dataset and data from the 2008 Wenchuan 36 earthquake aftershock confirm that the proposed UBDN method is very accurate and 37 has a lower rate of missed phase detection, outperforming solutions that adapt 38 traditional methods by an order of magnitude in terms of error percentage.

\section{Keywords}

40 U-net; Bidirectional Long Short Term Memory; Phase identification; Wenchuan 41 aftershocks 


\section{Introduction}

Seismic phase identification is a solid foundation for earthquake prevention and disaster reduction work (e.g., earthquake early warning systems). Previously, manual identification and travel timetable-based calculations were the two main methods used to analyze various seismic phase data and identify seismic phases. In recent years, with the growth in the number of seismic stations, the amount of seismic data available has also increased significantly. The efficiency and accuracy of manual identification has therefore gradually decreased. At the same time, the noise pollution caused by urbanization leads to higher rates of missed detection of low-level earthquakes. Manual identification can no longer maintain the accuracy and efficiency of phase detection. Seismic phase automatic identification is thus becoming a more widely used approach (Wu et al. 2004). It improves efficiency and reduces cost through faster processing of seismic data and higher identification rates.

Nowadays, seismic phase automatic detection is widely used to detect earthquakes. It utilizes characteristic equations, taking into account different parameters (e.g., amplitude and frequency). Thresholds are set based on these equations. Once a detected seismic phase reaches a preset threshold, the occurrence of an earthquake can be confirmed and its arrival time determined. Several methods are applied to seismic phase automatic identification. The Short-/Long-Term Average (STA/LTA) method uses the relationship between the average ratio of the short and the long time windows (Allen et al. 1978; Baer et al. 1987; Withers et al. 1998), and the threshold value. The Akaike 
information criterion (AIC) method is used to find the best division point (Akaike et al.

dimension method then uses the fractal dimension value to determine the first arrival

et al. 2017). These methods are affected by environmental noise and have poor stability,

resulting in low accuracy of identification and high rates of missed detection. In addition, the recognition results of these methods are significantly affected by the selection of features, functions, and parameters. The results often differ greatly from expectation when the signal-to-noise ratio (SNR) is low. Therefore, these methods are not efficient in seismic phase automatic identification when dealing with very large datasets. manage massive datasets (Dai et al. 1997). Artificial neural networks have been developed and widely applied to the automatic identification of seismic phases because of their strong learning ability. The size of the dataset caused by the increasing number of seismic stations can therefore be addressed by applying neural networks. When handling massive datasets, an artificial neural network requires both training and converging data and the determination of related parameters (e.g., neurons; layers)

81 through continuous experiment (Zhang et al. 1998; Wang et al. 2006; Wang et al. 2008). To some extent, the application of neural networks to the automatic identification of

83 seismic phases is limited due to the training processes required. 

solving the neural network's training difficulties (Ross et al. 2018). In particular, the

Convolutional Neural Network (CNN) approach to deep learning has been widely used in recent years. The $\mathrm{CNN}$ is classified according to multi-, shallow, and deep layers of convolutional structures. The multilayer convolution structure learns the features of the different levels, while the shallow layer learns the local features of the signal. Meanwhile, the deep convolutional layer is better at learning abstract features, which improves the classification and generalization capabilities of the CNN model compared to others. The deep convolutional layer structure of $\mathrm{CNN}$ is widely used in seismic signal noise reduction, seismic phase classification, and related matters (Zhao et al. 2019). Perol applied CNN to seismic phase identification (Perol et al. 2018), designing an eight-layer $\mathrm{CNN}$ with high identification accuracy. Ross manually annotated the dataset and used CNN for training (Ross et al. 2018). Experimental results have shown that this trained CNN model has high identification accuracy. Meanwhile, the identification effect with low SNR and different regions is still within the ideal range.

99 Yu Ziye input three-component seismic data into a 17-layer Inception model based on

100 CNN after high-pass filtering (Yu Ziye et al. 2018). At the same time, 100 datasets with 101 different degrees of noise were added and tested. Their results proved that the deep 102 learning method has a high tolerance for noise and is more stable than the AR103 AIC+STA/LTA method. However, the above models based on CNN still have the 104 problems of long training time and low computational efficiency. 
106 phase autoidentification has greatly improved the training speed (Zhao Ming et al.

107 2019), the accuracy of seismic phase identification, and the efficiency of dealing with

108 the continuous waveform, all in comparison with STA/LTA. However, the U-net based

109 training model still results in a 50\% missed detection rate. The U-net is known for its

110 efficient learning ability on two- or three-dimensional data. However, its high missed

111 detection rate leads to poor learning ability for one-dimensional time series data (e.g.,

112 seismic data). In contrast, the use of Bidirectional Long Short-Term Memory (Bi-

113 LSTM) in deep learning has proven to be efficient at processing the context of

114 sequences and is more suitable for one-dimensional time series data. The application of

115 Bi-LSTM to the identification and classification of heart sound signals of congenital

116 heart disease by Zhu Lili and the pattern identification of quality control graphs by $\mathrm{Wu}$

117 Changliang have proved its efficiency with such data (Zhu Lili. 2019; Wu et al. (2019)).

118 To address the limitations in the application of U-net models to seismic phase

119 autoidentification, we propose in this paper a novel U-net Bidirectional Long-Term

120 Memory Deep Network (UBDN). This combines the advantages of U-net (e.g., fast

121 training and accurate seismic phase identification) and Bi-LSTM (e.g., efficiency with

122 one-dimensional time series data). The UBDN specifically maintains the accuracy of

123 seismic phase identification. Based on that, a timing relationship can be established

124 between seismic phase features. Seismic phase autodetection can therefore be achieved

125 with high phase identification rates, low missed detection rates, and faster run times. 


\section{Methods}

Taking the U-net as the framework, the structure of the UBDN model is shown in

129 Fig.1. It can be seen that the input of the UBDN model is the original seismic waveform,

130 and the output is marked with the $\mathrm{P}$ and $\mathrm{S}$ wave phases for the input. The blue dashed

131 box contains the U-net component, and the red dashed box the Bi-LSTM network part.

132 The UBDN model uses the U-net to extract and learn the $\mathrm{P}$ and $\mathrm{S}$ wave phase

133 characteristics in the seismic waveform data, and then utilizes Bi-LSTM to identify the

$134 \mathrm{P}$ and $\mathrm{S}$ wave phases in the new seismic waveform.

135

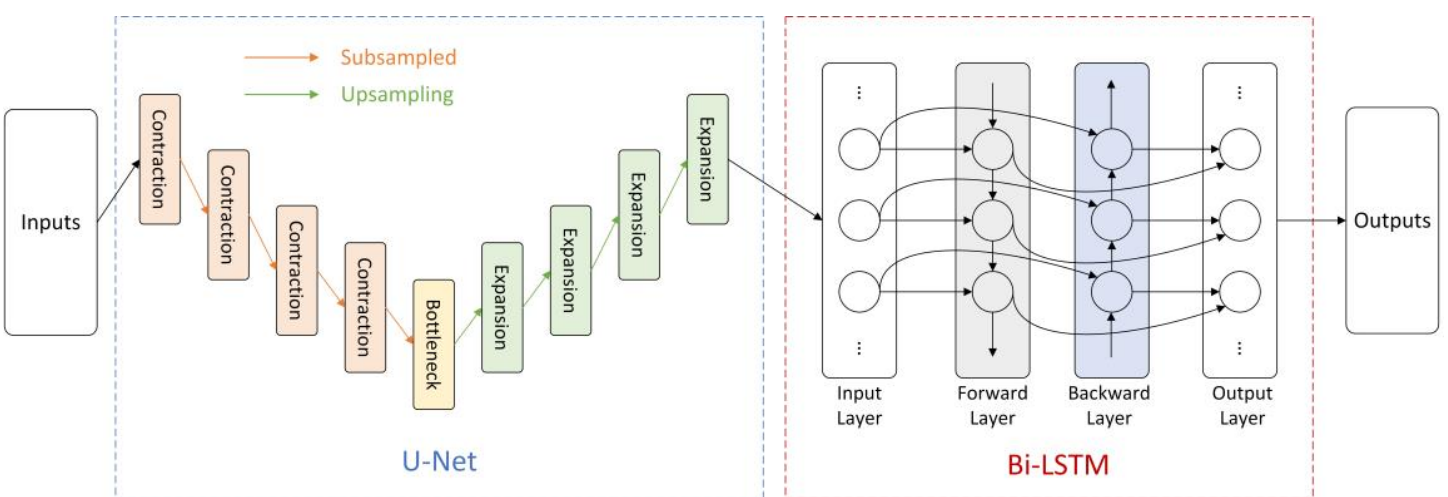

136

Fig.1 The UBDN model structure

137

The U-net realizes the extraction of the features in the seismic waveform by

138 determining the location of the $\mathrm{P}$ and $\mathrm{S}$ phases and finding the corresponding seismic

139 points. The U-net model structure is shown in Fig.2. The U-net consists of subsampling

140 and upsampling layers. The subsampling layer comprises 2 one-dimensional

141 convolutional layers and a pooling layer. In order to prevent overfitting, some dropout

142 layers were randomly added. The upsampling layer consisted of a transposed 
143 convolutional, cropping, and convolutional layer. A dropout layer was again randomly

144 added to prevent overfitting. Compared with the classic U-net, the network input was

145 processed for dimensionality reduction. The input of the U-net defaults to two- or three-

146 dimensional image data, and the seismic waveform data were one-dimensional time

147 series data. Therefore, all inputs and outputs inside the network needed to be reduced

148 to one dimension. The four subsampling layers on the left side performed convolution

149 and pooling operations to extract the abstract features of the seismic phase; whereas the

150 four upsampling layers on the right side completed operations such as transposed

151 convolution and connection between the left and right symmetric layers. Recovering

152 the detailed features of the seismic phases based on the learned features on the left side

153 was conducive to solving the problem of seismic phase classification. Finally, the

154 probability value of $\mathrm{P}$ wave, $\mathrm{S}$ wave, or noise was calculated by the activation function,

155 and the category of the sampling point determined by comparing with the set threshold.

156 At the same time, a loss function was used to adjust the hyperparameters such as the

157 weight and bias of each layer to minimize the difference between the current network's

158 predicted value and the target value. 


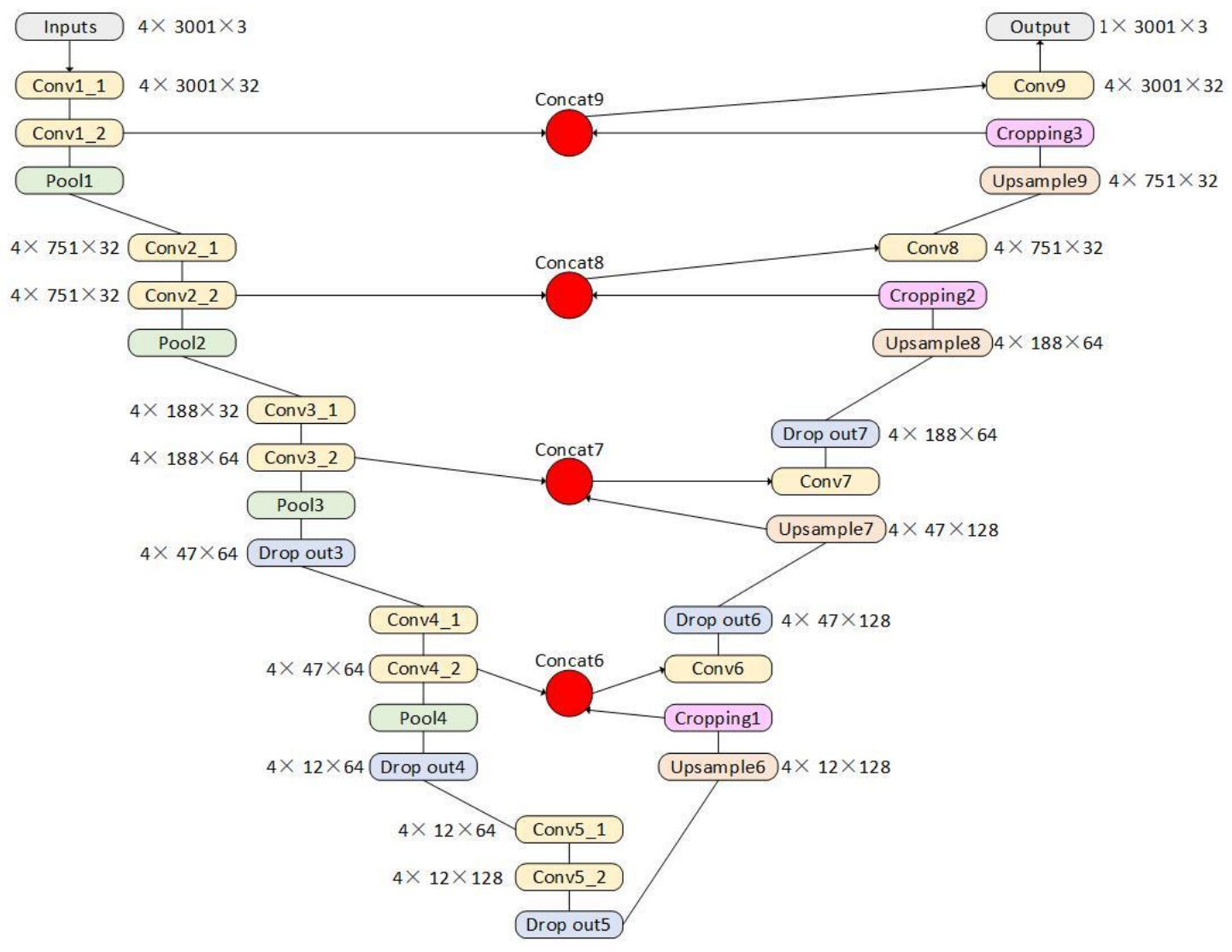

161 The loss function used in this model is shown in formula (1):

$$
\text { Loss }=-\sum_{i=1}^{3} \sum_{j=1}^{n} Y_{i j}^{\prime} \cdot \log \left(Y_{i j}\right)
$$

163 In formula (1), $Y^{\prime}$ is a label using binary coding; $i=1,2,3$ respectively 164 represent noise, $\mathrm{P}$ phase, and $\mathrm{S}$ phase; $n$ is the number of waveform sampling points; 165 and $j=1, \ldots, n$ is the sampling point number. $Y^{\prime}$ is shown in formula (2):

$$
Y_{i}^{\prime}=\left\{\begin{array}{l}
Y_{1}^{\prime}:[1,0,0] \\
Y_{2}^{\prime}:[0,1,0] \\
Y_{3}^{\prime}:[0,0,1]
\end{array}\right.
$$




$$
Y_{i}=\frac{e^{z_{i}}}{\sum_{k=1}^{3} e^{z_{k}}}
$$

In formula (3), $z$ is the output tensor ([m, n, 3]) of the last layer, and $m$ is the

171 number of input data.

173 both a forward and a reverse LSTM. The forward LSTM obtains a sequence $h_{a}$

174 according to normal input. The reverse LSTM reverses the input, and then passes it

175 through a network with the same structure as the forward LSTM but with different

176 weight parameters. Using this approach we eventually obtained a sequence which we

177 then reversed to obtain $h_{b}$. Finally, we added the two sequences to get $H$, which is the

178 final result through the Bi-LSTM network, as shown in formula (4).

$$
H=h_{a} \oplus h_{b}
$$

180

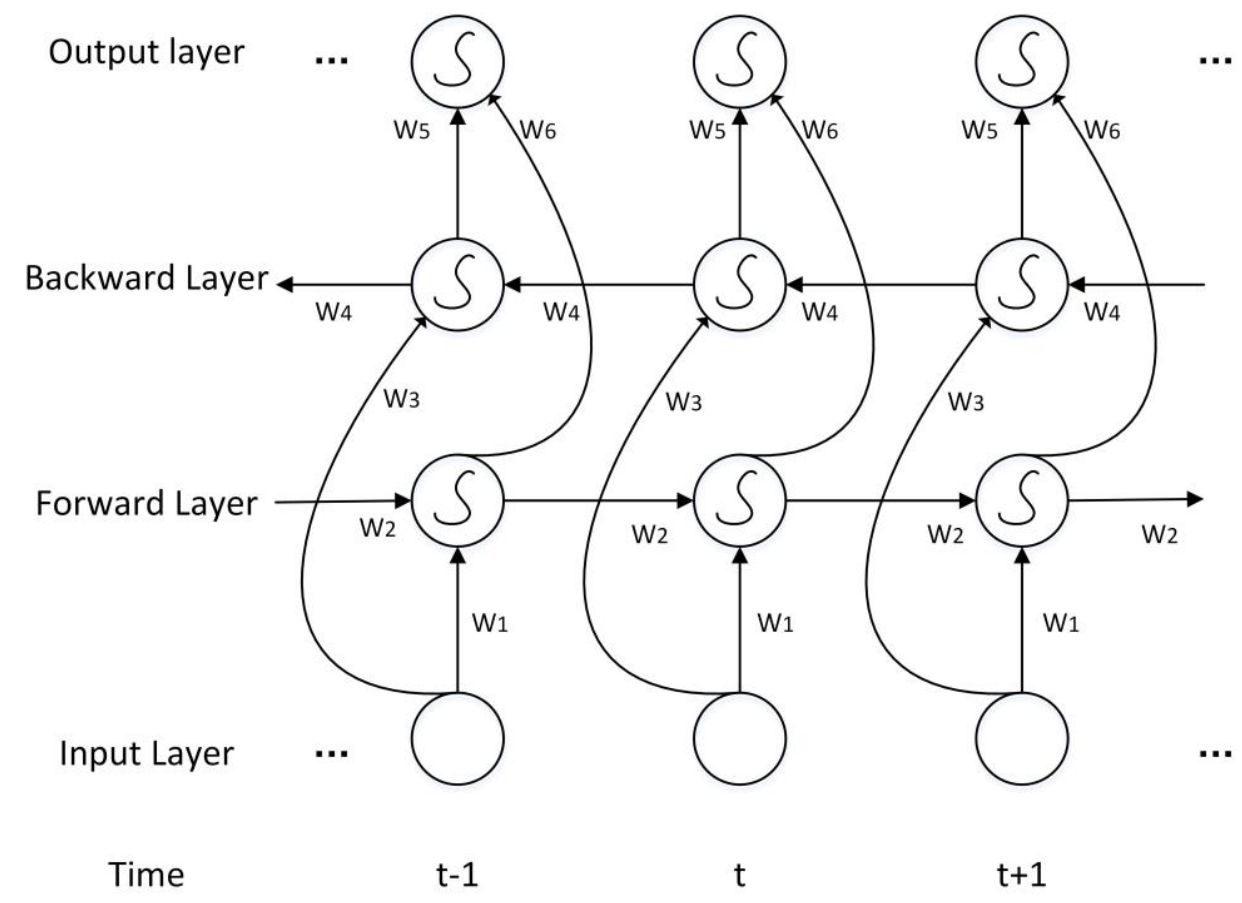


184 in the sequence, divide the sequence information into two directions for input into the model, use two hidden layers to store the input information in both directions, and connect the corresponding outputs of the hidden layers to the same output layer. Both of them have the same structures and are independent of each other, and they only accept different sequence inputs. Therefore, the final hidden layer contains the positive and negative time series data for the dataset. Utilizing the advantages of Bi-LSTM in processing time series data, this establishes the timing relationship between seismic phase features, which solves the problem of missed detection caused by the use of the U-net in seismic phase identification.

\section{Data and evaluation indicators}

\subsection{Data preprocessing}

The UBDN model requires a large number of preset samples for training. The sample data used here came from the Stanford Earthquake Dataset (STEAD), which is sourced mainly from the International Seismological Center, the National Earthquake Information Center, the Northern California Seismic Network, the Southern California Seismic Network, the Pacific Northwest Seismic Network, The New Madrid Seismic Network, the Incorporated Research Institutions for Seismology, the Advanced National Seismic System Composite Catalog, and the Global Seismograph Network. The STEAD comprises data recorded by seismic stations around the world from January 1984 to August 2018 (Mousavi et al. 2019). This article mainly selected seismic 
events of a magnitude between M0.5 and 2.5 with a SNR between 20 and $30 \mathrm{~dB}$; the sampling rate was $100 \mathrm{~Hz}$ for sample data.

The sample data were preprocessed and the waveforms from $3 \mathrm{~s}$ before the arrival of the $\mathrm{P}$ wave to $10 \mathrm{~s}$ after the arrival of the $\mathrm{S}$ wave selected. We then uniformly cut the sample data to a length of 30s (assigned a zero value if the length is insufficient), then normalized all the data as shown in formula (5), where $v_{i}$ represents the amplitude value of the seismic event signal, and data enhancement operations such as translation, noise addition, and filtering are adopted (Hayakawa et al.1995; Schroff et al. 2015).

$$
v_{i}=\frac{v_{i}}{\max \left|v_{i}\right|}
$$

To ensure the quality of the dataset, it was manually cleaned and obvious labeling errors corrected. The final sample dataset consisted of 43,700 items, of which $80 \%$ were used as training data and $20 \%$ as testing data.

\subsection{Evaluation index}

In order to objectively evaluate the performance of the UBDN model in practical applications and ensure the reliability of the experimental results, we used the calculation of the root mean square error (RMSE), accuracy (A), and missed detection rate $(\mathrm{M})$ of the different methods. The three formulas used to calculate these indicators were as follows:

$$
\begin{gathered}
\text { RMSE }=\sqrt{\frac{1}{n} \sum_{i=1}^{n}\left[\left(T_{i}-t_{i}\right)^{2}\right]} \\
A=\frac{T_{e}}{\left(T_{e}+F_{e}\right)}
\end{gathered}
$$




$$
M=\frac{F_{n}}{\left(T_{e}+F_{n}\right)}
$$

RMSE denotes the root mean square error between the calculated time $T_{i}$ of the of the model, and the preprocessed dataset divided into the training and testing datasets

244 according to the 80:20 ratio given above. (2) Model training, where the relevant dataset 
245 was used to train the UBDN model, the U-net to learn the seismic phase features, and

246 the Bi-LSTM then used the features to complete the phase identification of the time

247 series signal and output the seismic waveform data of the marked phase. (3) Model

248 evaluation and comparison, in which we used the output model to compare the pickup

249 and reference times, and calculated the RMSE, accuracy (A) and missed detection rate

250 (M) to evaluate the effectiveness of the model's seismic phase identification.

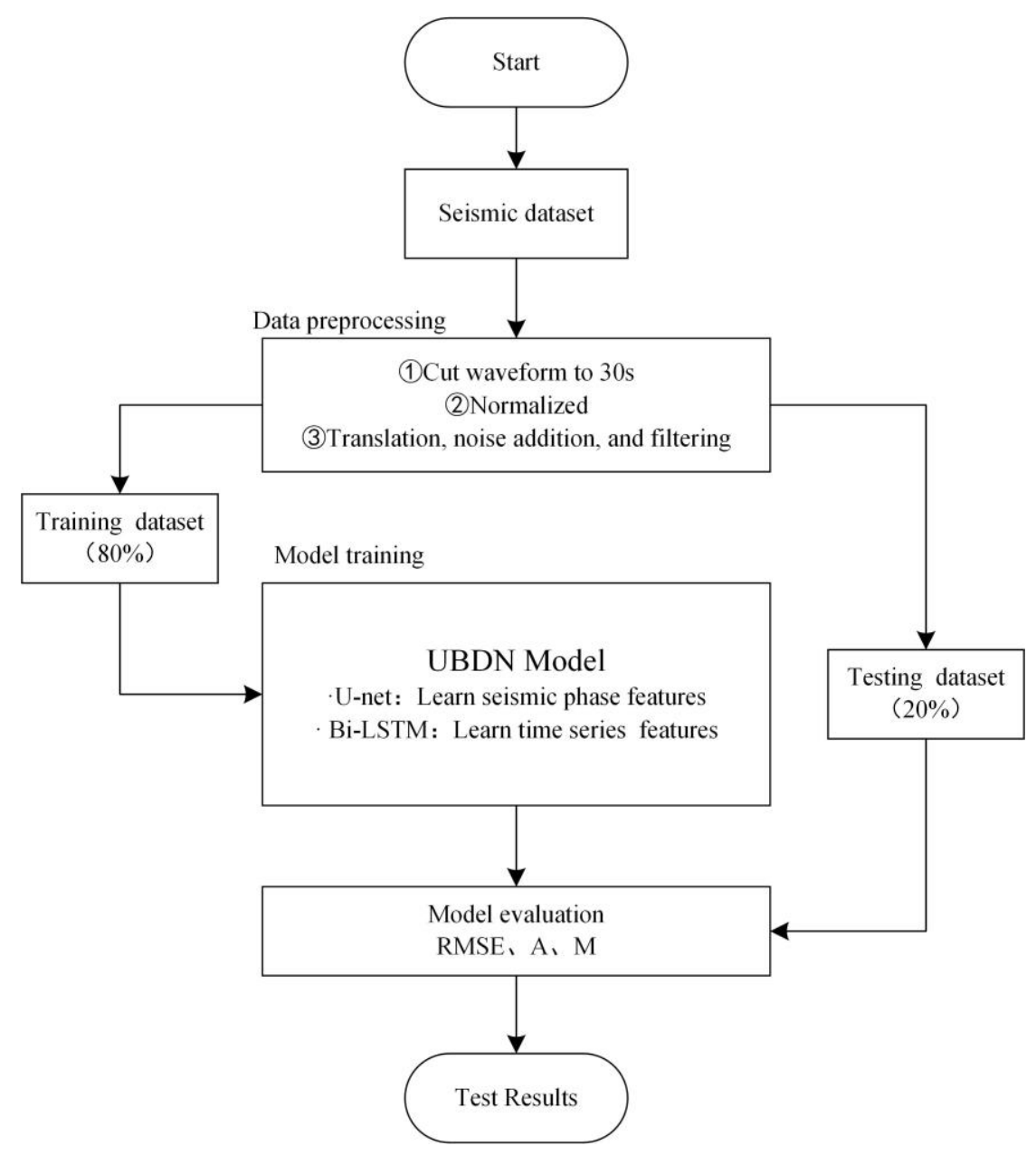


261 of the model because it has the advantages of high computational efficiency and adaptive learning rate. L2 regularization was used to prevent overfitting (Kingma et al. 263 2014).

265 the training accuracy of the model; Fig. 5(b) its loss function value; Fig. 5(c) the phase 266 identification accuracy (A) of the P wave on the testing dataset; and Fig. 5(d) the phase 267 identification accuracy (A) of the $\mathrm{S}$ wave on the testing dataset. It can be seen from 268 Fig.s 5(a) and 5(b) that the UBDN model had an accuracy (A) of $98 \%$ after training the 269 fourth cycle (about 20,000 time steps), and the loss value dropped to 0.03 . The accuracy 270 (A) and loss values of the following five cycles remained at this level, which shows that 271 the UBDN model converged at this point after about 45,000 time steps. Fig.s 5(c) and 272 5(d) show that the accuracy (A) of the $\mathrm{P}$ and $\mathrm{S}$ wave phase identification on the testing 273 dataset increased over time. From time steps 10,000 to 45,000 , the P and $\mathrm{S}$ waves 274 increased from $65.1 \%$ and $43.7 \%$ to $90.1 \%$ and $89.5 \%$ respectively. This indicates that 
275 the model did not overfit; furthermore, the longer the training time, the higher the 276 accuracy. The experimental results in Fig. 5 show that the accuracy (A) of phase 277 identification of $\mathrm{P}$ and $\mathrm{S}$ waves by the model was $90.1 \%$ and $89.5 \%$, respectively, which 278 meets the accuracy requirements for seismic phase identification. This indicates the 279 model can be used effectively for this purpose.

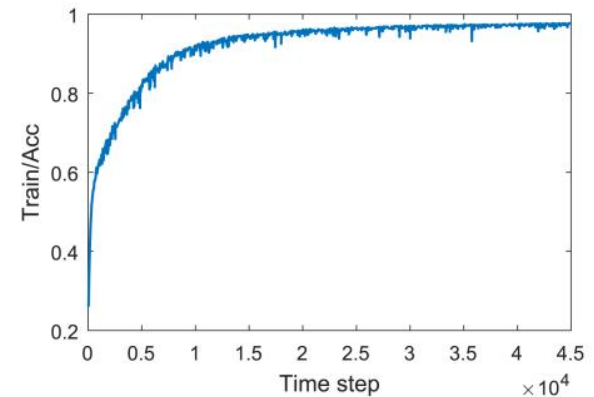

(a)

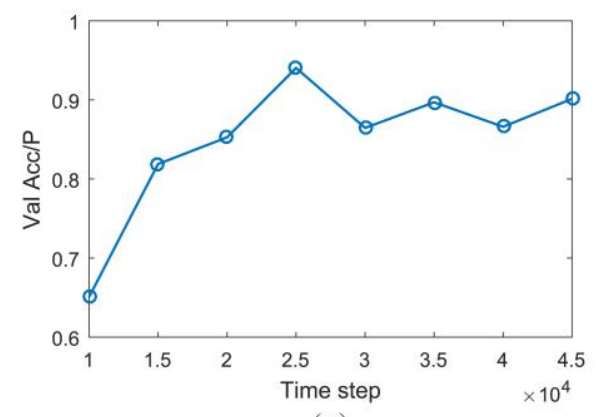

(c)

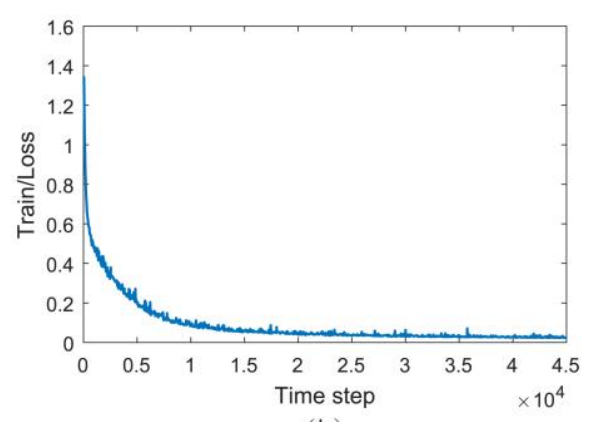

(b)

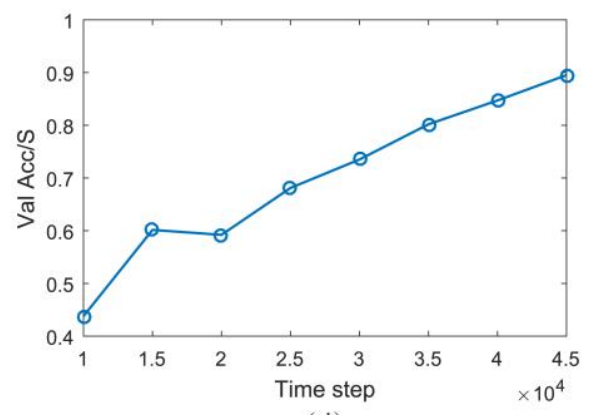

(d)

Fig. 5 Training and testing process of the UBDN model

\subsection{Comparative Experiments}

In order to verify the seismic phase identification ability of the UBDN model, we

284 compared it with the deep learning U-net [19], STA/LTA, and AIC seismic phase 285 identification methods. In the comparative experiments, all models used a unified 286 dataset of 2,000 pieces of waveform, and the SNR was concentrated between 20 30dB.

287 We used formulas (6), (7), and (8) to calculate the RMSE, accuracy (A), and missed 
detection rate $(\mathrm{M})$ so as to evaluate the detection results of the four models. The

289 experimental results are shown in Table 1.

290 Table 1 - Comparison of the UDBN model with the U-net, STA/LTA, and AIC

methods

\begin{tabular}{|c|c|c|c|c|}
\hline \multicolumn{2}{|c|}{ Method } & \multirow{2}{*}{$\begin{array}{c}\text { RMSE } \\
0.26\end{array}$} & \multirow{2}{*}{$\begin{array}{c}\text { A } \\
87.99 \%\end{array}$} & \multirow{2}{*}{$\begin{array}{c}\text { M } \\
11.45 \%\end{array}$} \\
\hline \multirow{2}{*}{ UBDN } & $\mathbf{P}$ & & & \\
\hline & $\mathbf{S}$ & 0.27 & $87.60 \%$ & $14.58 \%$ \\
\hline \multirow{2}{*}{ U-net } & $\mathbf{P}$ & 0.35 & $81.26 \%$ & $30.74 \%$ \\
\hline & $\mathbf{S}$ & 0.41 & $83.49 \%$ & $28.40 \%$ \\
\hline \multirow{2}{*}{ STA/LTA } & $\mathbf{P}$ & 0.49 & $73.45 \%$ & $37.52 \%$ \\
\hline & $\mathbf{S}$ & 0.62 & $76.23 \%$ & $35.87 \%$ \\
\hline \multirow{2}{*}{ AIC } & $\mathbf{P}$ & 0.43 & $75.13 \%$ & $32.81 \%$ \\
\hline & $\mathbf{S}$ & 0.58 & $78.92 \%$ & $30.72 \%$ \\
\hline
\end{tabular}

As can be seen from Table 1, the RMSE of the UBDN model for the $\mathrm{P}$ and $\mathrm{S}$ waves

293 was $0.26 \mathrm{~s}$ and $0.27 \mathrm{~s}$, and the accuracy (A) was $87.99 \%$ and $87.61 \%$. This represents a

294 significant improvement over the traditional STA/LTA and AIC methods. The UBDN

295 model also shows improvement in comparison with the U-net model. This indicates the

296 UBDN model has obvious advantages compared with other methods in terms of

297 accuracy. In terms of missed detection rates, the parameter M for the UBDN model was

$29811.45 \%$ and $14.58 \%$. Compared with other methods, this is a significant reduction and 
299 indicates that the combination of the U-net and Bi-LSTM network approaches has 300 achieved an ideal result in reducing the missed detection rate. The three evaluation 301 indicators were highest for the UBDN model among the four methods tested.

Fig. 6 shows the various possible scenarios for the UBDN model in the phase

303 identification process. In the Fig., the black line shows when the $\mathrm{P}$ wave is picked up

304 manually, and the red line when the $S$ wave is picked up manually. Fig. 6(a) shows the

305 correct detection of the P and S wave phases; Fig. 6(b) the correct detection of the P-

306 wave phase and the incorrect detection of the S-wave phase; Fig. 6(c) shows the S-wave

307 phase detected and the P-wave phase; Fig. 6(d) shows the reverse scenario.
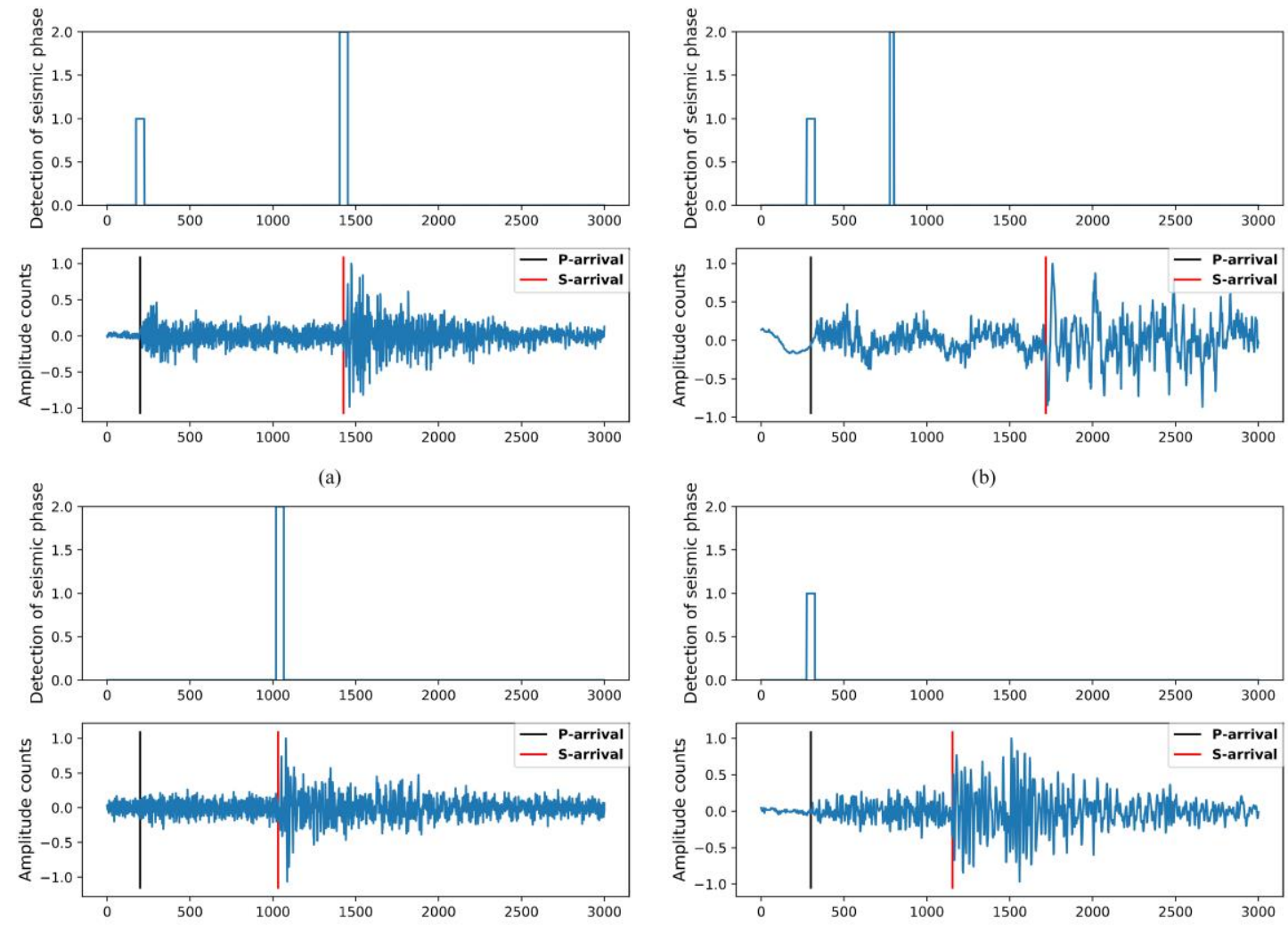

(c)

(d)

Fig. 6 Different phase identification scenarios for the UBDN model 
312 earthquake event, it was applied to the aftershock dataset of the Wenchuan earthquake

313 from May to September 2008. This dataset contains both seismic events and phase data.

314 A total of 2,026 phase data items were collected from 76 stations. The SNR of the data

315 was concentrated between $2 \sim 5 \mathrm{~dB}$, the sampling rate was $200 \mathrm{~Hz}$, and the attribute fields

316 included the earthquake time and longitude, latitude, depth, magnitude type, magnitude,

317 reference location, event type, and so on (Data sharing infrastructure of national

318 earthquake data center - http://data.earthquake.cn). An example event is shown in Fig.

319 7. This depicts the seismic events taking place at $14: 53$ on May 12, 2008. The

320 magnitude is Ms6.3; the focal depth $14 \mathrm{~km}$ as recorded by the Maoxin Nanxin station

321 (51MXN); the black line denotes the arrival of the manually picked $\mathrm{P}$ wave; and the red

322 line the arrival of the manually picked S wave. Fig.s 7(a), 7(b), and 7(c) represent the

323 vertical, north-south, and east-west seismic waveforms, respectively. The UBDN

324 model was used to identify the phases of the Wenchuan earthquake aftershock dataset.

325 The results are shown in Table 2. 


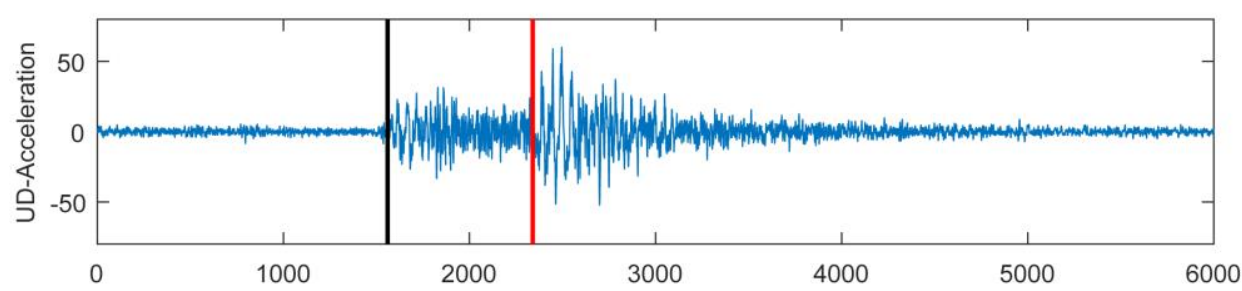

(a)

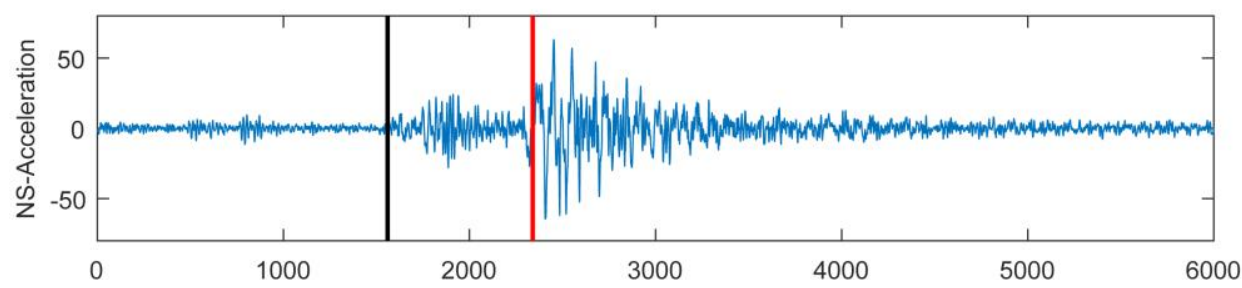

(b)

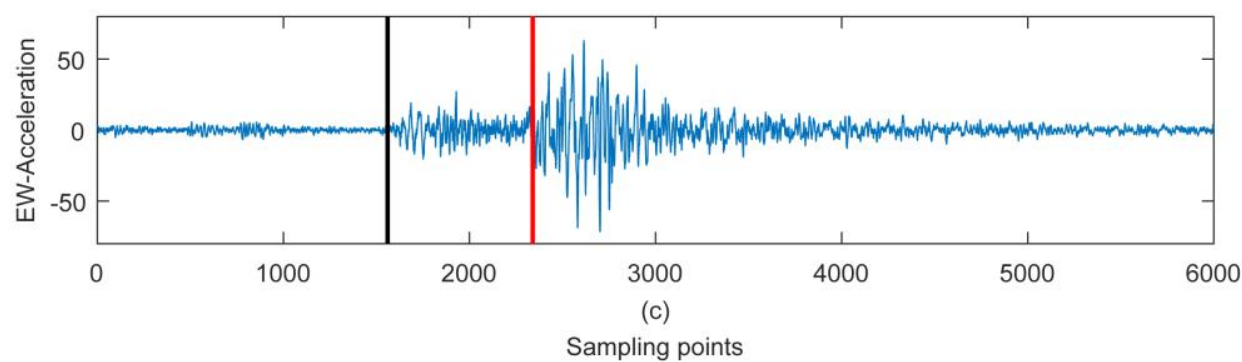

Fig. 7 Examples of seismic event waveforms earthquake aftershock dataset

\begin{tabular}{|c|c|c|c|c|}
\hline \multicolumn{2}{|c|}{ Method } & RMSE & A & M \\
\hline \multirow{2}{*}{ UBDN } & P & 0.31 & $80.69 \%$ & $17.18 \%$ \\
\cline { 2 - 5 } & S & 0.34 & $77.87 \%$ & $20.56 \%$ \\
\hline
\end{tabular}

It can be seen from Table 2 that the RMSE of the UBDN model on the Wenchuan

331 earthquake aftershock dataset was $0.31 \mathrm{~s}$ and $0.34 \mathrm{~s}$, the accuracy (A) $80.69 \%$ and $33277.87 \%$, and the missed detection rate (M) $17.18 \%$ and $20.56 \%$. Compared with the 333 experimental results of the testing dataset, the effect of the UBDN model on the 
334 Wenchuan earthquake aftershock dataset is slightly poorer. This may be attributable to

335 the different SNR of the datasets. To verify this hypothesis, the SNR of the aftershock

336 dataset was concentrated in the range $2 \sim 5 \mathrm{~dB}$, and the SNR of the testing dataset in the

337 range 20 30dB. Phase identification tests were then performed on five datasets with

338 different SNR ranges of 0 10dB, 10 20 dB, 20 30 dB, 30 40 dB, and >40 dB, with

339 the number of events in each dataset set as 1,000 .

340 The results of this experiment are shown in Fig. 8. It can be seen that the RMSE,

341 accuracy (A), and missed detection rate (M) all have a linear relationship with SNR. As

342 the SNR increases, RMSE and M gradually decrease, but A gradually rises. This

343 indicates that the higher the SNR of the data, the better the seismic phase identification

344 of the UBDN model. Although the effect for the actual Wenchuan earthquake

345 aftershock dataset is slightly lower than that for the testing dataset, it can still meet the

346 needs of practical applications. Therefore, the UBDN model has a certain degree of

347 adaptability, and can still achieve a good seismic phase identification effect under the 348 condition of low SNR. 


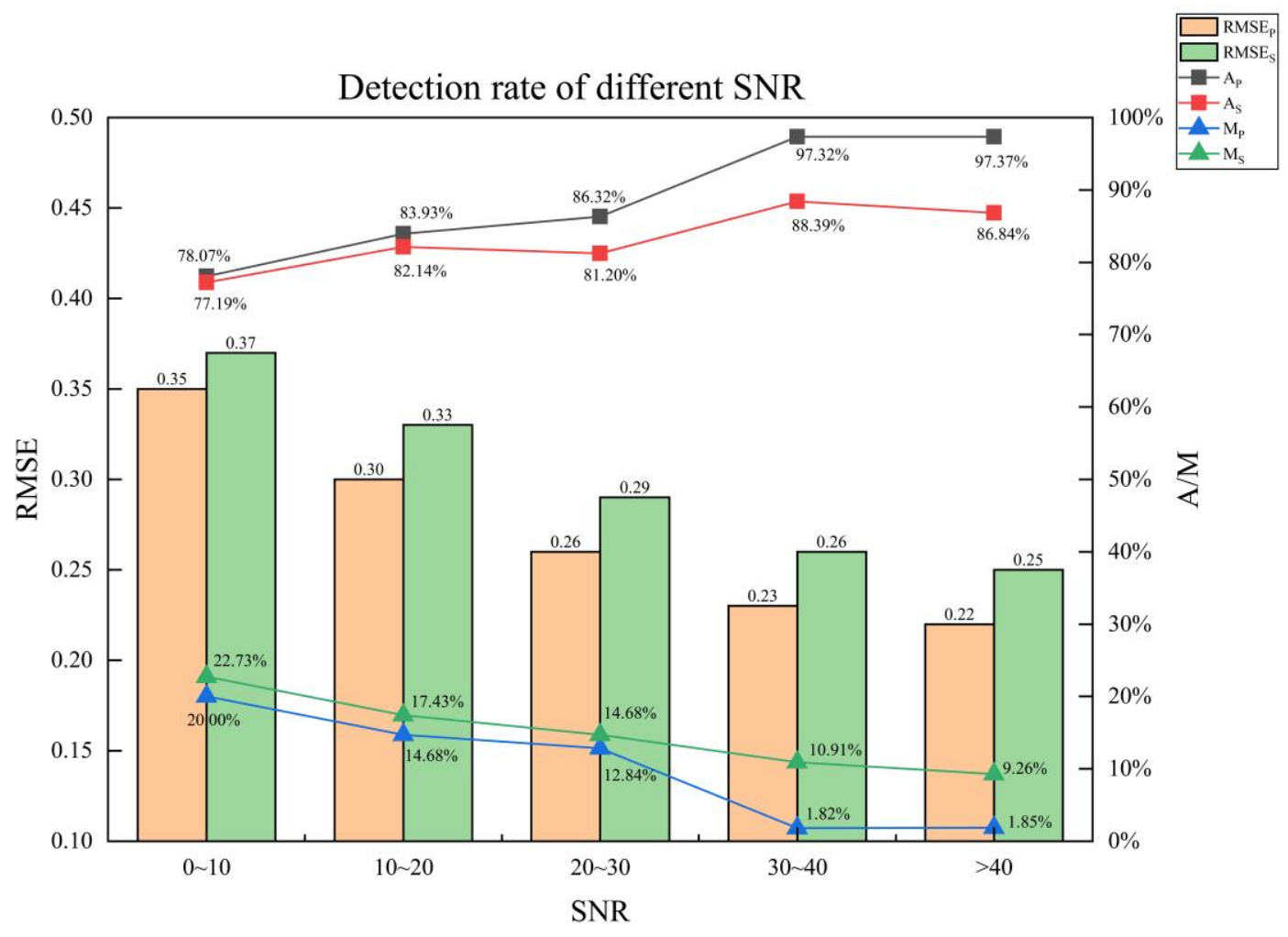

Fig. 8 Phase identification results for datasets with different SNR

\section{Conclusion}

Based on U-net and Bi-LSTM structures, we designed a UBDN seismic phase

353 automatic identification model, used it to train a dataset, and established the relationship

354 between the phase arrival time and the seismic waveform phase for real-time

355 identification. By comparing the results of the trained model with those for other

356 identification methods, and performing testing and experimental analysis using the

3572008 Wenchuan earthquake aftershock dataset, we can draw the following conclusions.

358 Firstly, the UBDN model can achieve sufficient accuracy to meet the requirements of

359 seismic phase identification; secondly, compared with other methods, it offers a

360 significant improvement on accuracy and a significant reduction in the missed detection 
361 rate; and thirdly, using the UBDN model with a real-life dataset (i.e., the Wenchuan

362 earthquake aftershock data) shows that there is still a strong phase identification effect

363 and further indicates that the model has good adaptability.

364 In practical applications, low-SNR data have an impact on the identification

365 capabilities of the UBDN model. Future research may consider using seismic data

366 collected by the same type of instrument and processed by the same noise reduction

367 method as a dataset for model training, according to the noise level and sensitivity of

368 seismic instruments and the difference in data noise reduction methods. This approach

369 can greatly reduce the influence of SNR on the results of the UBDN model training and

370 identification process and thus improve its stability.

371

372 Declarations

373 Ethics approval and consent to participate

374 Not applicable.

375 Consent for publication

376 Not applicable.

377 Availability of data and materials

378 The datasets used and/or analyzed during the current study are available from the

379 corresponding author upon reasonable request. 
381 The authors declare no conflict of interests.

\section{Funding}

383 This work was supported by the Special Fund of Fundamental Scientific Research

384 Business Expense for Higher School of Central Government [no. ZY20180111], The

385 National Key Research and Development Programme of China (Grant No 386 2018YFC1503801), and the Scientific Research Project Item of Hebei Province 387 Education Department (Grant No.QN2018317).

388 Authors' contributions

389 Jianxian Cai, Zhitao Gao contributed to the conception of the study; Jianxian Cai, Xun

390 Dai performed the experiment; Xun Dai, Zhitao Gao contributed significantly to 391 analysis and manuscript preparation; Jianxian Cai, Xun Dai performed the data analyses 392 and wrote the manuscript; Jianxian Cai, Zhitao Gao, Yan Shi helped perform the 393 analysis with constructive discussions.

\section{Acknowledgements}

395 Acknowledgement for the data support from " China Earthquake Networks Center,

396 National Earthquake Data Center. (http://data.earthquake.cn)"; Acknowledgement for 397 the data support from " the Stanford Earthquake Dataset (STEAD). 398 (https://github.com/smousavi05/STEAD)". 


\section{References}

400 Akaike H (1974) A new look at the statistical model identification. IEEE Transactions 401 on Automatic Control 19(6): 716-723.

402 Akazawa T (2004) A technique for automatic detection of onset time of P-and S-phases 403 in strong motion records.In:The 13th World Conference on Earthquake Engineering: 404 International Association of Earthquake Engineering, Vancouver BC, Canada, January 4052004.

406 Allen R V (1978) Automatic earthquake recognition and timing from single traces.

407 Bulletin of the Seismological Society of America 68(5): 1521-1532.

408 Baer M, Kradolfer U ( 1987) An automatic phase picker for local and teleseismic events. 409 Bulletin of the Seismological Society of America 77(4): 1437-1445.

410 Boschetti F, Dentith M D, List R D, (1996) A fractal-based algorithm for detecting first 411 arrivals on seismic trances. Geophysics 61(4): 1095-1102. 412 https://doi.org/10.1190/1.1444030

413 Cao Maosen, Ren Qingwen, Wan Linmei, Luo Yong (2004) Length Picking up first 414 arrival of seismic wave with fractal dimension algorithm.Petroleum geophysical 415 exploration (05): 510-514.

416 Chang Xu, Liu Yike (2002) Generalized fractal dimension of seismic records and its 417 application.Acta geophysica Sinica (06): 839-846. doi:10.3321/j.issn:0001$418 \quad 5733.2002 .06 .011$ 
419 Dai H, MacBeth C (1997) The application of back-propagation neural network to 420 automatic picking seismic arrival from single-component recordings. Journal of 421 Geophysical Research: Solid Earth (1978-2012) 102(B7): 15105-15113.

422 Han Xiaojun, Shi zejin, Li Yalin (2002) An improved algorithm for picking up the first 423 arrival of seismic wave using fractal dimension.Petroleum geophysical exploration 424 37(1): 60-63. doi:10.3321/j.issn:1000-7210.2002.01.012

425 Hayakawa, Marumoto, Sawada (1995) Effects of the chaotic noise on the performance 426 of a neural network model for optimization problems. Physical review. E, Statistical 427 physics, plasmas, fluids, and related interdisciplinary topics 51(4): R2693-R2696. 428 https://doi.org/10.1103/PhysRevE.51.R2693

429 Kingma D. P, Ba J, Adam (2014). A method for stochastic optimization. arXiv preprint 430 arXiv:1412.6980.

431 Liu Jinsong, Wang Yun, Yao Zhenxing (2013) Automatic picking up method of 432 microseismic signals when they arrive. Acta geophysica Sinica 56(5): 1660-1666, 433 doi:10.6038/cjg20130523. doi:10.6038/cjg20130523

434 Mousavi S M, Sheng Y, Zhu W, et al. (2019). Stanford Earthquake Dataset (STEAD): 435 A global dataset of seismic signals for AI. IEEE Access: 179464-179476. doi: 436 10.1109/ACCESS.2019.2947848

437 Perol T, Gharbi M, Denolle M A ( 2018) Convolutional neural network for earthquake 438 detection and location. Science Advances 4(2). doi: 10.1126/sciadv.1700578 
439 Ross Z E, Meier M A, Hauksson E (2018) P wave arrival picking and first-motion 440 polarity determination with deep learning. Journal of Geophysical Research: Solid 441 Earth 123(6): 5120-5129. https://doi.org/10.1029/2017JB015251

442 Ross Z E, Meier M A, Hauksson E, Heaton T H (2018) Generalized seismic phase 443 detection with deep learning. Bulletin of the Seismological Society of America 108(5A): 444 2894-2901. https://doi.org/10.1785/0120180080

445 Schroff F, Kalenichenko D, Philbin J (2015) FaceNet: A unified embedding for face 446 recognition and clustering. 2015 IEEE Conference on Computer Vision and Pattern 447 Recognition (CVPR).Boston, MA, USA: IEEE: 815-823.

448 Sleeman R, Van Eck T (1998) Robust automatic P-phase picking: an on-line 449 implementation in the analysis of broadband seismogram recordings. Physics of the 450 Earth and Planetary Interiors 113(1-4): 265-275. https://doi.org/10.1016/S0031$451 \quad 9201(99) 00007-2$

452 Tang Yalei (2017) Application of fractal theory in P-wave phase identification. 453 Dissertation, Southwest Jiaotong University.

454 Wang Ji, Chen Jiuhui (2008) Automatic detection of seismic events using artificial 455 neural network. Seismogeomagnetic observation and research (03): 41-45. 456 doi:10.3969/j.issn.1003-3246.2008.03.007 
457 Wang Jinfeng, Luo Shengxian (2006) BP improvement of neural network and its 458 application in first break picking.Calculation technology of geophysical and 459 geochemical exploration (01): 14-17. doi:10.3969/j.issn.1001-1749.2006.01.005

460 Withers M, Aster R, Young C,Beiriger J,Harris M,Moore S,Trujillo J (1998) A 461 comparison of select trigger algorithms for automated global seismic phase and event 462 detection. Bulletin of the Seismological Society of America 88(1): 95-106.

463 Wu Changliang, Zhu Bo, Wan Yuwei, Zhao Shengran (2019) Pattern recognition of 464 quality control chart based on Bi LSTM. Software 40(07): 89-95. 465 doi:10.3969/j.issn.1003-6970.2019.07.016

466 Wu Dongpo (2004) Research on real time method of seismic phase identification. 467 Dissertation, Institute of engineering mechanics, China Seismological Bureau.

468 Yu Ziye,Chu Risheng,Sheng Minhan (2018) Deep neural network picking up P and s 469 arrival time of earthquake. Acta geophysica Sinica 61(12): 4873-4886. 470 doi:10.6038/cjg2018L0725

471 Zhang Fanmin, Li Qinghe, Zhang Yuansheng, Sheng Guoying, Fan Bing (1998) Using 472 artificial neural network theory to identify seismic signal and seismic phase[J].Journal 473 of Northwest seismology (04): 43-49.

474 Zhao Ming, Chen Shi, Fang Lihua, Yuen D A (2019) Study on the method of seismic 475 phase identification and arrival time picking based on U-shaped convolution neural network. Acta geophysica Sinica 62(08): 3034-3042. doi:10.6038/cjg2019M0495 
477 Zhao Ming,Chen Shi, Yuen D (2019) Automatic classification and recognition of 478 seismic waveform based on deep learning convolutional neural network.Acta 479 geophysica Sinica 62(1): 374-382. doi:10.6038/cjg2019M0151.

480 Zhu Lili (2019) Recognition of congenital heart disease based on Bi LSTM network. 481 Dissertation, Yunnan University.

482 483 
Figures

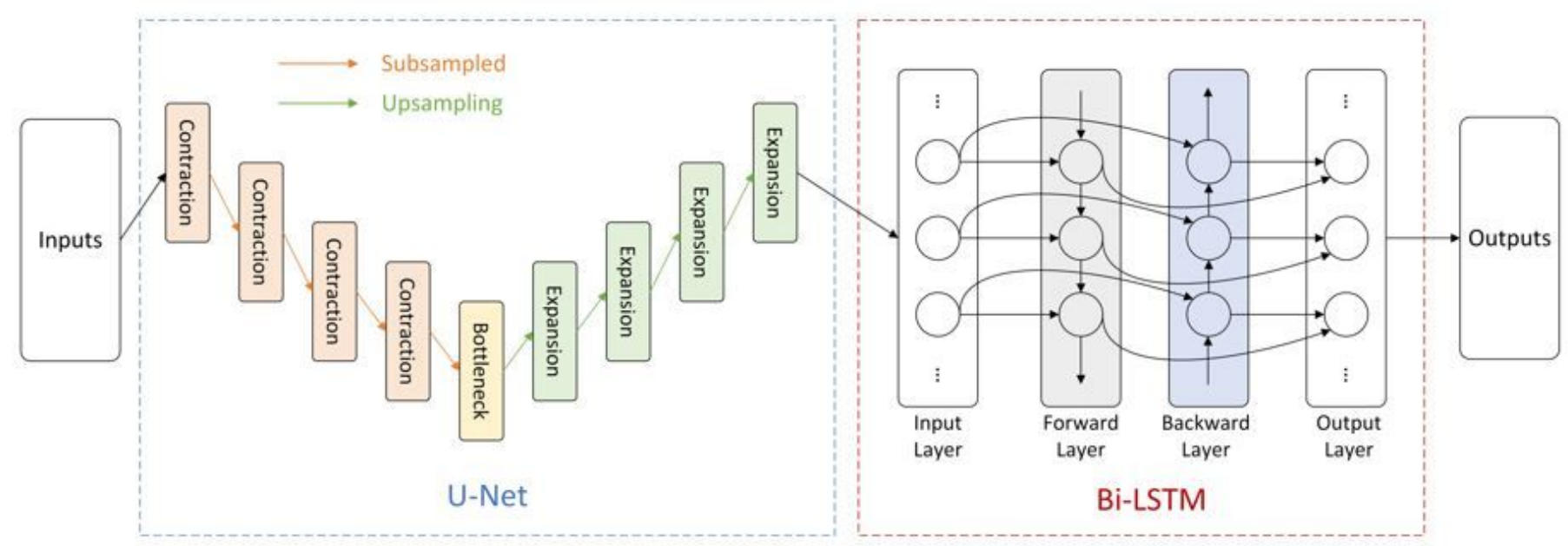

Figure 1

The UBDN model structure 


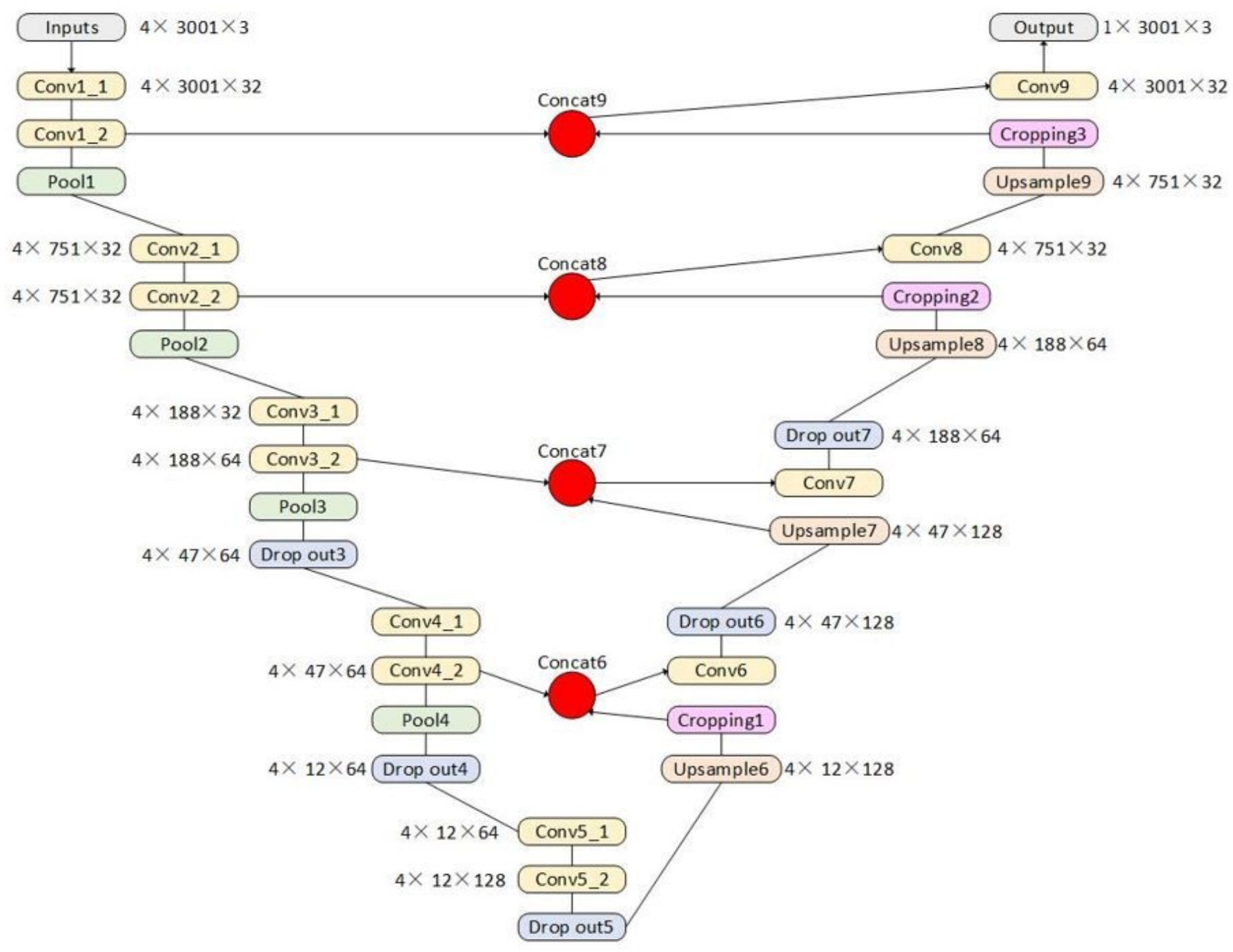

Figure 2

The U-net model structure 


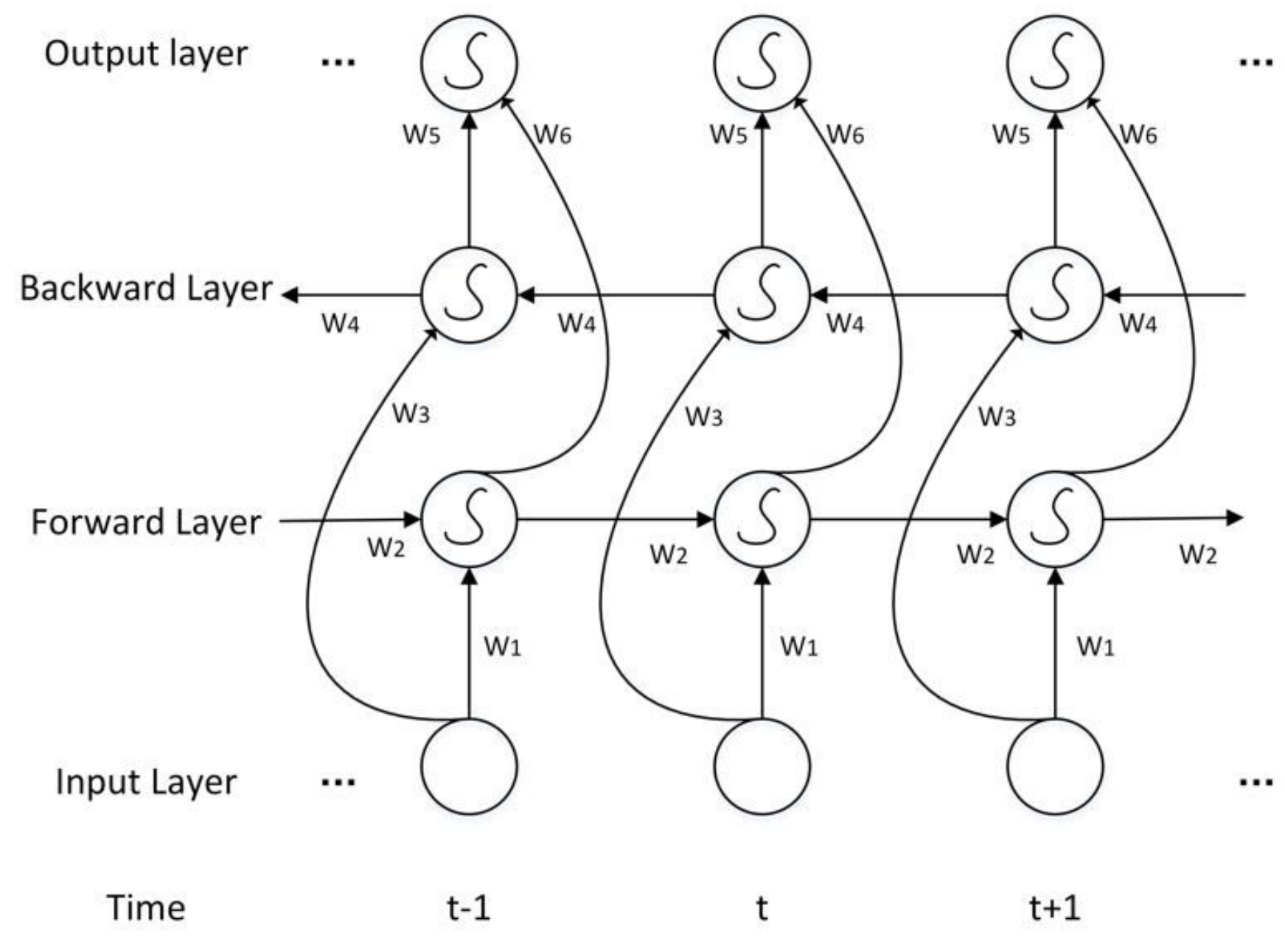

Figure 3

The Bi-LSTM model structure 


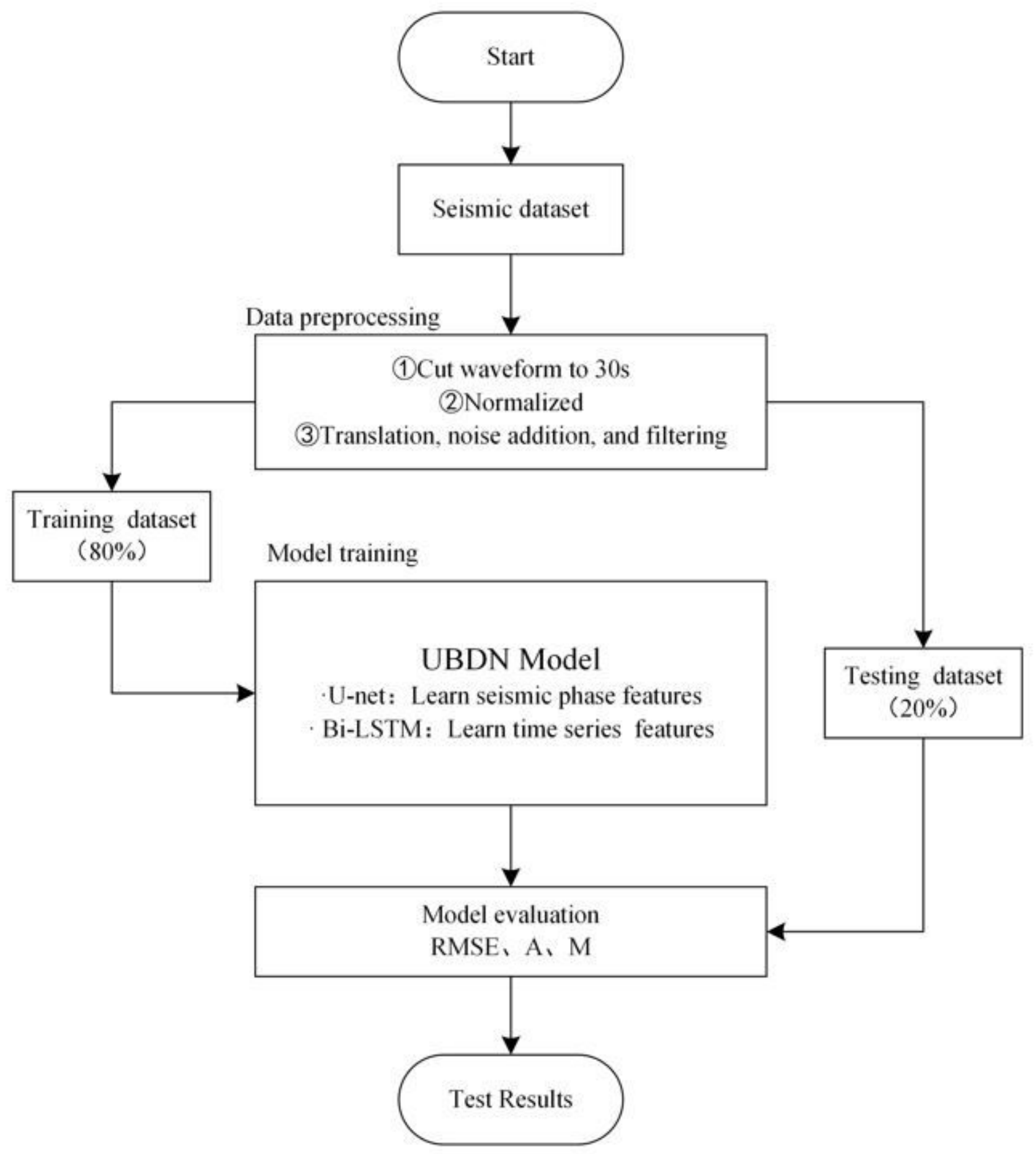

Figure 4

Experimental flowchart 


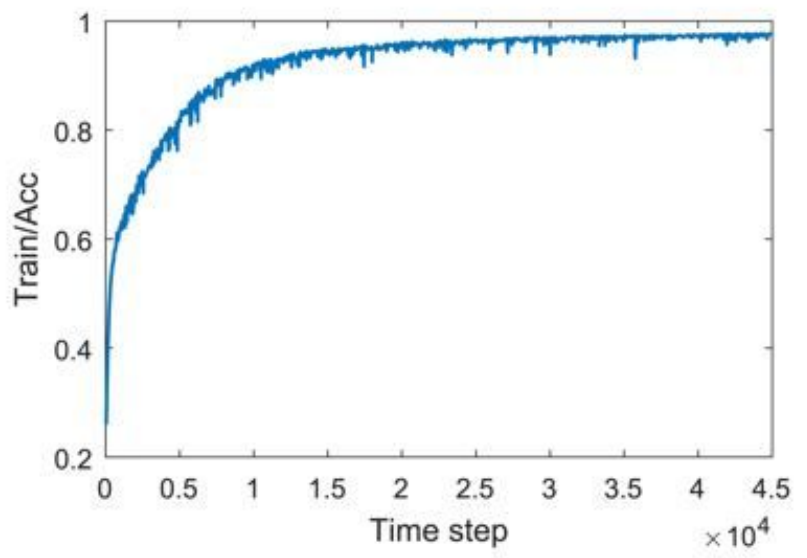

(a)

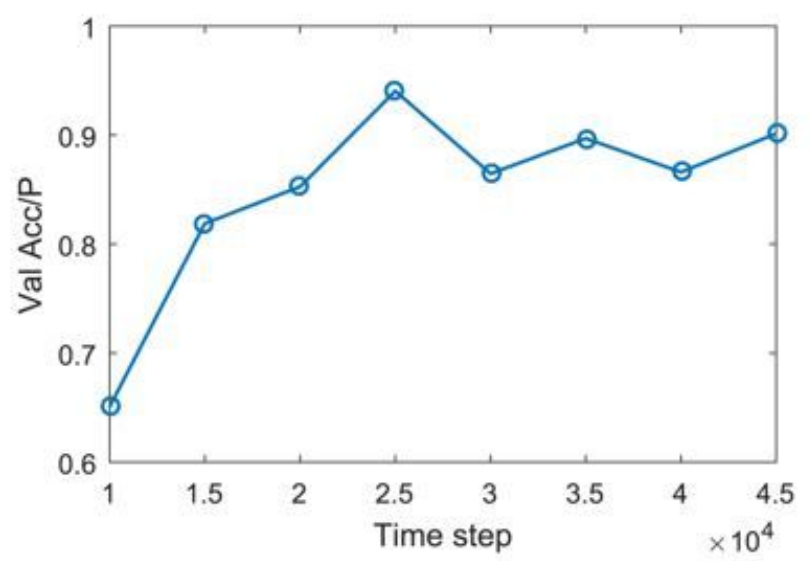

(c)

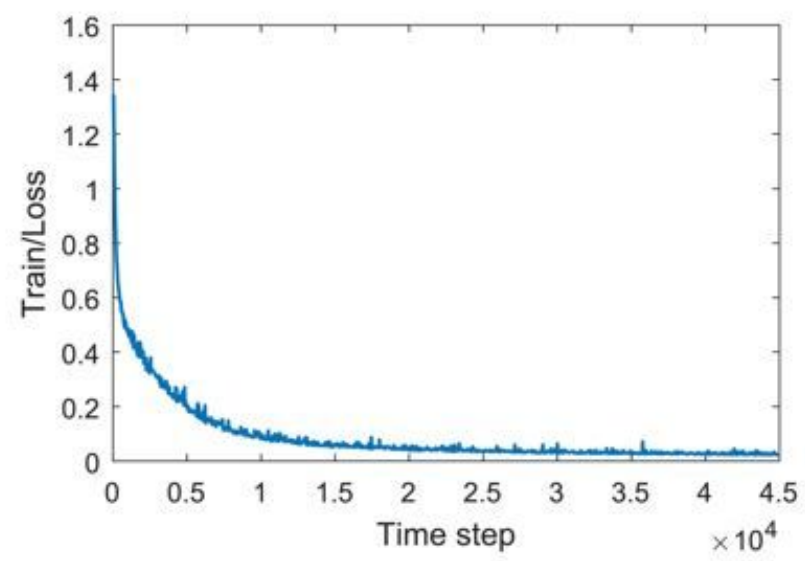

(b)

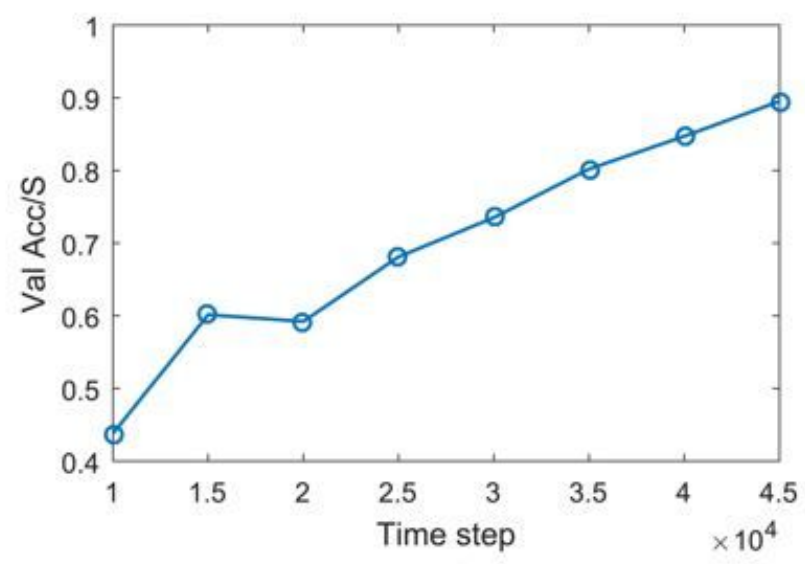

(d)

\section{Figure 5}

Training and testing process of the UBDN model 

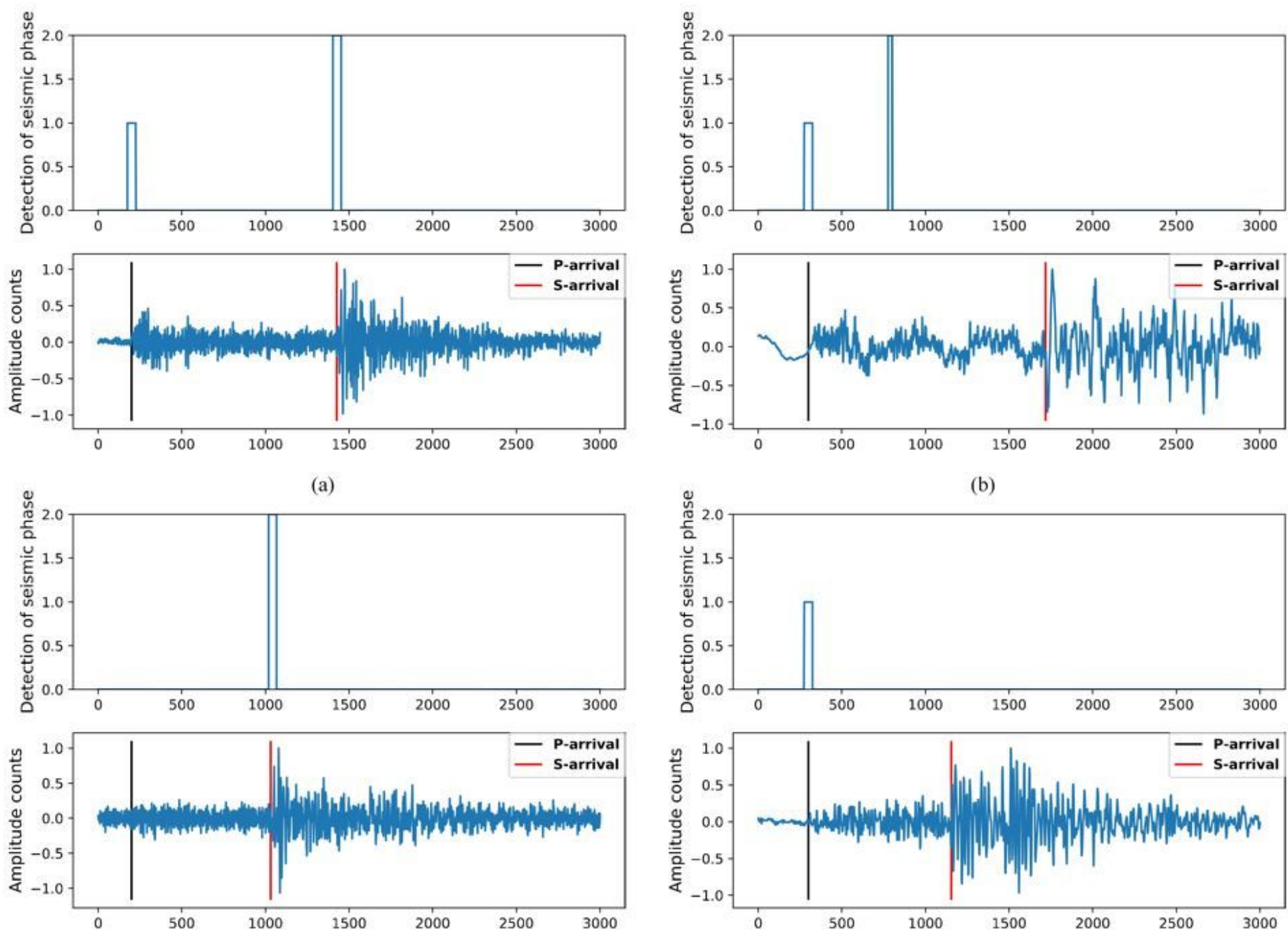

(c)

(d)

Figure 6

Different phase identification scenarios for the UBDN model 


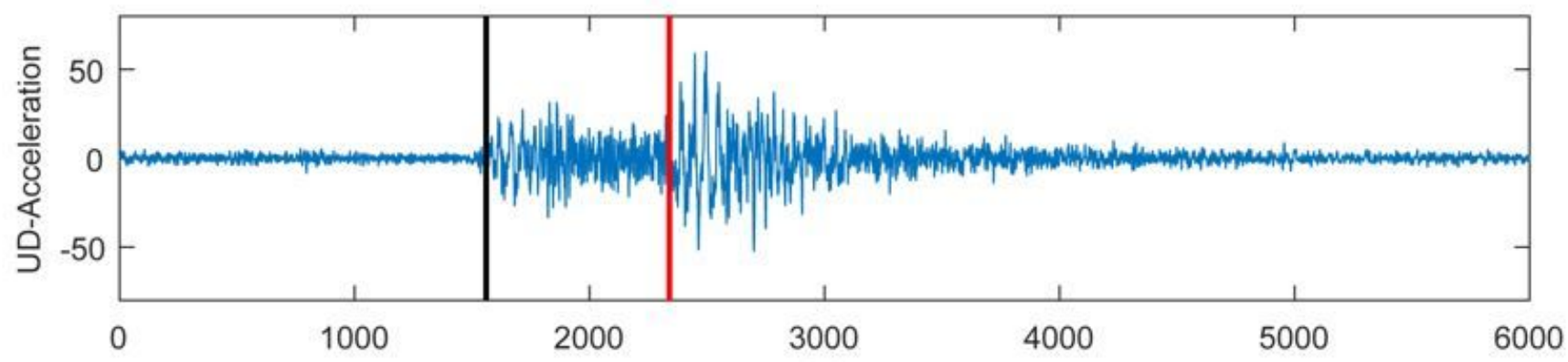

(a)

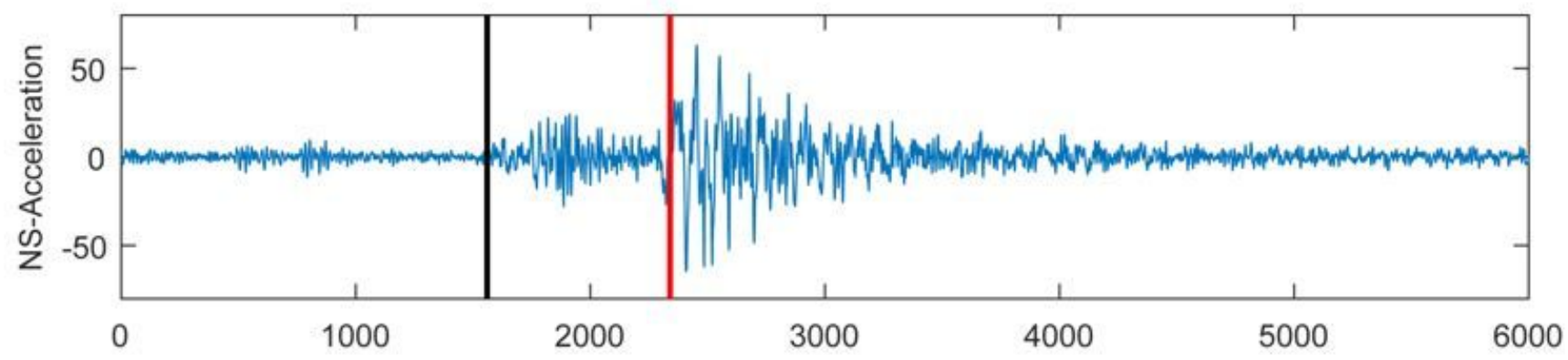

(b)

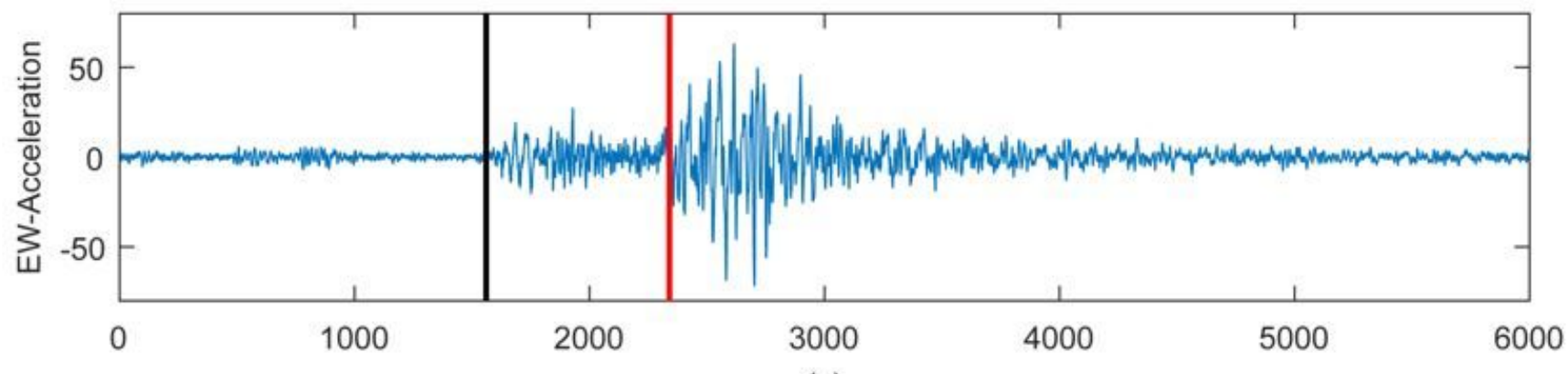

(c)

Sampling points

Figure 7

Examples of seismic event waveforms 


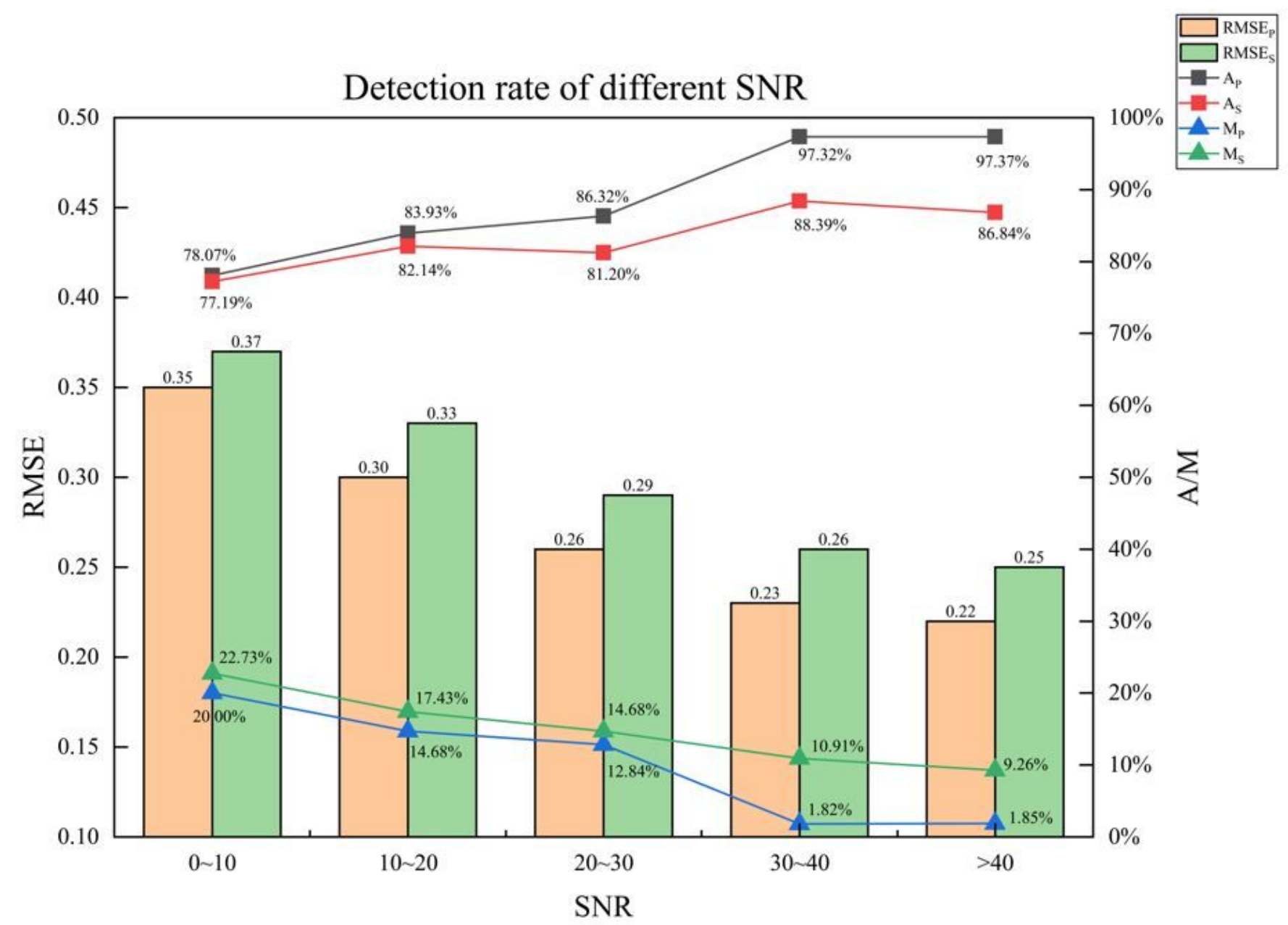

Figure 8

Phase identification results for datasets with different SNR

\section{Supplementary Files}

This is a list of supplementary files associated with this preprint. Click to download.

- Graphicalabstract.png 